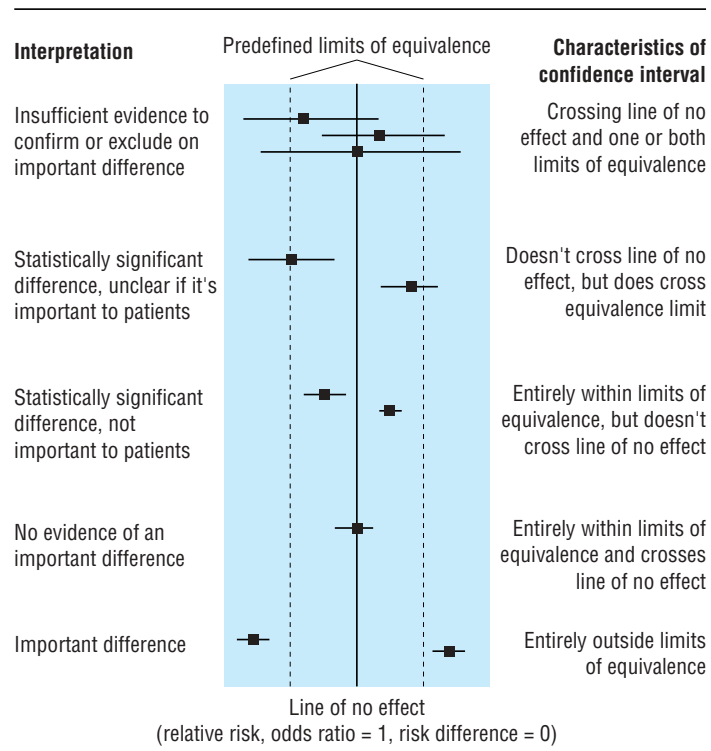

Relation between confidence interval, line of no effect, and thresholds for important differences (adapted from Armitage, Berry, and Matthews $\left.{ }^{4}\right)$

When is it reasonable to claim that a study has proved that no effect or no difference exists? The correct answer is "never," because some uncertainty will always exist. However, we need to have some rules for deciding when we are fairly sure that we have excluded an important benefit or harm. This implies that some threshold must be decided, in advance, for what size of effect is clinically important in that situation. This concept is not new and is used in designing equivalence studies, which set out to show whether one intervention is as good as another. ${ }^{3}$ Thresholds, often called limits of equivalence, are set between which an effect is designated as being too small to be important. Outcomes of, for example, studies of effectiveness can then be related to these thresholds. This is shown in the figure, where the confidence interval from a study is interpreted in the context of predefined limits of equivalence.

Of course, setting such thresholds is not straightforward. How big a reduction in the incidence of HIV-1 infection is important? How large an increase in incidence is important? Who should decide? How different should the thresholds be for different groups of patients and different outcomes? These are difficult questions, and although we may not be able to find easy answers to them, we can at least be more explicit in reporting what we have found in our research. Wording such as "our results are compatible with a decrease of this much or an increase of this much" would be more informative.

What can we do to help ensure that in another decade we will be closer to heeding the advice of Altman and Bland? Firstly, considering results of a particular study in the context of all available research which considers the same question can increase statistical power, reduce uncertainty, and thus reduce the confusing reporting of underpowered studies. Such an approach might have clarified the implications of a recent study of passive smoking published in the $B M J .^{5}$ Secondly, researchers need to be precise in their interpretation and language and avoid the temptation to save words by reducing the summary of the study to such an extent that the correct meaning is lost. Thirdly, journals need to be willing to publish uncertain results and thus reduce the pressure on researchers to report their results as definitive. ${ }^{6}$ We need to create a culture that is comfortable with estimating and discussing uncertainty.

Phil Alderson associate director

UK Cochrane Centre, Oxford OX2 7LG (palderson@cochrane.co.uk)

I thank Iain Chalmers and Mike Clarke for comments on draft versions.

Competing interests: None declared.

1 Altman DG, Bland JM. Absence of evidence is not evidence of absence. BMJ 1995;311:485.

2 Kamali A, Quigley M, Nakiyingi J, Kinsman J, Kengeya-Kayondo J, Gopal $\mathrm{R}$, et al. Syndomic management of sexually-transmitted infections and behaviour change interventions on transmission of HIV-1 in rural Uganda: a community randomised trial. Lancet 2003;361:645-52.

3 Greene WL, Concato J, Feinstein AR. Claims of equivalence in medical Greene WL, Concato J, Feinstein AR. Claims of equivalence in medical
research: are they supported by the evidence? Ann Intern Med research: are they

4 Armitage P, Berry G, Matthews JNS. Statistical methods in medical research. 4th ed. Oxford: Blackwell Science, 2002

5 Enstrom JE, Kabat GC. Environmental tobacco smoke and tobacco related mortality in a prospective study of Californians, 1960-98. BMJ 2003;326:1057-60.

6 Alderson P, Roberts I. Should journals publish systematic reviews that find no evidence to guide practice? Examples from injury research. $B M J$ 2000;320:376-7.

\title{
Aspirin resistance
}

\section{May be a cause of recurrent ischaemic vascular events in patients taking aspirin}

$\mathrm{A}$ spirin reduces the odds of serious atherothrombotic vascular events and death in a broad category of high risk patients by about one quarter. ${ }^{1}$ The primary antithrombotic mechanism is believed to be inhibition of the biosynthesis of thromboxane (and thus platelet activation) by inactivation of platelet cyclo-oxygenase- 1 . However, aspirin is not that effective. It still fails to prevent most (at least 75\%) serious vascular events in patients with symptomatic patients taking aspirin ("aspirin treatment failures") have many possible causes (box), and aspirin resistance has emerged as an additional contender. ${ }^{23}$

But what is aspirin resistance? Aspirin resistance has been used to describe several different phenomena. One is the inability of aspirin to protect patients from ischaemic vascular events. This has also been called clinical aspirin resistance. ${ }^{4}$ However, this definition is non-specific and could apply to any of the conditions listed in the box. Furthermore, it is not real- 


\section{Editorials}

\begin{tabular}{|c|c|c|c|}
\hline Test & Method & Advantages & Limitations \\
\hline \multirow[t]{2}{*}{ Platelet aggregation } & Optical platelet aggregation & $\begin{array}{l}\text { Widely available } \\
\text { Correlated with clinical events }\end{array}$ & $\begin{array}{l}\text { Not specific } \\
\text { Uncertain sensitivity } \\
\text { Labour intensive } \\
\text { Operator and interpreter dependent }\end{array}$ \\
\hline & $\begin{array}{l}\text { Semi-automated platelet aggregometry } \\
\text { (PFA-100, Ultegra RPFA) }\end{array}$ & $\begin{array}{l}\text { Simple } \\
\text { Rapid }\end{array}$ & $\begin{array}{l}\text { Not specific } \\
\text { Uncertain sensitivity } \\
\text { Uncertain correlation with clinical events }\end{array}$ \\
\hline Bleeding time & Skin bleeding time & $\begin{array}{l}\text { Simple } \\
\text { Widely available }\end{array}$ & $\begin{array}{l}\text { Not specific } \\
\text { Not sensitive } \\
\text { Operator dependent } \\
\text { Limited reproducibility } \\
\text { Uncertain correlation with clinical events }\end{array}$ \\
\hline Thromboxane generation & Urinary thromboxane excretion & Correlated with clinical events & $\begin{array}{l}\text { Uncertain specificity } \\
\text { Uncertain sensitivity } \\
\text { Uncertain reproducibility } \\
\text { Not widely evaluated }\end{array}$ \\
\hline
\end{tabular}

PFA=platelet function analyser; RPFA=rapid platelet function analyser.

istic to expect that all vascular complications can be prevented by any single preventive strategy. ${ }^{5}$ Aspirin resistance has also been used to describe an inability of aspirin to produce an anticipated effect on one or more tests of platelet function, such as inhibiting biosynthesis of thromboxane, ${ }^{6}$ inhibiting platelet aggregation, ${ }^{7}$ and causing a prolongation of the bleeding time..$^{5}$ This has been called biochemical aspirin resistance. ${ }^{4}$ However, the precise qualitative and quantitative abnormalities of platelet function which define biochemical aspirin resistance have not been established, let alone their clinical relevance. As shown in the table, there are several different laboratory tests of platelet function which are being used to diagnose "biochemical" aspirin resistance, and each has its own limitations.

For a laboratory measure of biochemical aspirin resistance to have clinical utility it must be associated independently and consistently with the occurrence of recurrent vascular events in patients taking aspirin; it must be standardised and valid; and clinical management should be altered on the basis of the results of testing-for example, it should be shown in randomised controlled trials that reversing the laboratory abnormality (with treatment) is followed by a reduction in the incidence of recurrent vascular events while taking aspirin. Finally, the overall benefits of testing should outweigh any adverse consequences and costs. ${ }^{9}$

Two recent studies meet the first criterion, but no study meets the other three criteria. The first study showed an independent and significant association between increasing baseline urinary concentrations of 11-dehydrothromboxane B2 (a marker of in vivo thromboxane generation) and an increasing risk of future myocardial infarction or cardiovascular death in patients at high vascular risk who were treated with aspirin. ${ }^{6}$ The second study showed an independent and significant association between the failure of aspirin to suppress agonist induced platelet aggregation and an increasing risk of serious vascular events in 326 patients with coronary or cerebral vascular disease who were treated with aspirin (hazard ratio 4.1, 95\% confidence interval 1.4 to 12.1$).{ }^{7}$ In this study platelet aggregation was measured by optical platelet aggregometry. These data also show that up to $20 \%$ of future serious vascular events in high risk vascular patients may be attributable to a failure of aspirin to suppress thromboxane production or platelet aggregation. $^{67}$

The therapeutic implications of a valid and reliable screening test for aspirin resistance, coupled with an

Possible causes of recurrent ischaemic vascular events among patients taking aspirin

Non-atherothrombotic causes of vascular events

- Embolism from the heart (red, fibrin thrombi;

vegetations; calcium; tumour; prostheses)

- Arteritis

Reduced bioavailability of aspirin

- Inadequate intake of aspirin (poor compliance)

- Inadequate dose of aspirin

- Concurrent intake of certain non steroidal anti-inflammatory drugs (for example ibuprofen, indomethacin), possibly preventing the access of aspirin to cyclo-oxygenase- 1 binding site

Alternative pathways of platelet activation

- Platelet activation by pathways that are not blocked by aspirin (for example, red cell induced platelet activation: stimulation of collagen, adenosine diphosphate, epinephrine, and thrombin receptors on platelets)

- Increased platelet sensitivity to collagen and adenosine diphosphate

- Biosynthesis of thromboxane by pathways that are not blocked by aspirin (for example, by

cyclo-oxygenase-2 in monocytes and macrophages, and vascular endothelial cells)

Increased turnover of platelets

- Increased production of platelets by the bone marrow in response to stress (for example, after coronary artery bypass surgery), introducing into blood stream newly formed platelets unexposed to aspirin during the 24 hour dose interval (aspirin is given once daily and has only a 20 minute half life)

\section{Genetic polymorphisms}

- Polymorphisms involving platelet glycoprotein $\mathrm{Ia} / \mathrm{IIa}, \mathrm{Ib} / \mathrm{V} / \mathrm{IX}$, and $\mathrm{Ilb} / \mathrm{III}$ a receptors, and collagen and von Willebrand factor receptors

- Polymorphisms of cyclo-oxygenase-1,

cyclo-oxygenase-2, thromboxane $\mathrm{A}_{2}$-synthase, or other arachidonate metabolism enzymes

- Factor XIII Val34Leu polymorphism, leading to variable inhibition of factor XIII activation by low dose aspirin 
effective treatment, are exciting. However, before aspirin resistance can be accepted as a valid clinical entity worthy of screening and treatment, the other criteria mentioned above must be met. The first step is to develop a standardised definition and test of aspirin resistance. An appropriate definition of aspirin resistance may be: the lack of anticipated response to a therapeutic dose of aspirin (75-150 mg per day for at least five days in a compliant patient) that can be demonstrated by a specific, valid, and reliable laboratory measure of the antiplatelet effects of aspirin and which correlates significantly, independently, and consistently with an increased incidence of atherothrombotic vascular events. The definition may be refined in the future to include proven genetic determinants (for example, platelet polymorphisms), which mediate aspirin resistance and risk of ischaemic events. Further studies are required to externally validate the promise of urinary 11-dehydrothromboxane $\mathrm{B} 2$ and optical platelet aggregometry as laboratory measures of "clinical" aspirin resistance.

1 Antithrombotic Trialists' Collaboration. Collaborative meta-analysis of randomised trials of antiplatelet therapy for prevention of death, myocardial infarction, and stroke in high risk patients. BMJ 2002;324:71-86.

2 Helgason CM, Bolin KM, Hoff JA, Winkler SR, Mangat A, Tortorice KL, et al. Development of aspirin resistance in persons with previous ischaemic stroke. Stroke 1994;25:2331-6.

3 Gum PA, Kottke-Marchant K, Poggio ED, Gurm H, Welsh PA, Brooks L, et al. Profile and prevalence of aspirin resistance in patients with cardiovascular disease. Am J Cardiol 2001;88:230-5.

Bhatt DL, Topol EJ. Scientific and therapeutic advances in antiplatelet therapy. Nature Rev 2003;2:15-28.

5 Patrono $C$. Aspirin resistace: definition, mechanisms and clinicat read-outs. J Thromb Haemost 2003;1:1710-3.

Eikelboom JW, Hirsh J, Weitz JI, Johnston M, Yi Q Yusuf S. Aspirin resistance and the risk of myocardial infarction, stroke, or cardiovascular death
While awaiting the development of a reliable test and effective treatment for aspirin resistance, the most efficient strategy for clinicians to prevent aspirin failure is to make sure that the index event was atherothrombotic in origin, use an appropriate dose of aspirin (75$150 \mathrm{mg}$ daily), maintain a high level of compliance, and avoid combining aspirin with drugs such as ibuprofen that may reduce its effectiveness for the prevention of atherothrombotic vascular events..$^{10} 11$

\section{Graeme J Hankey consultant neurologist}

Stroke Unit, Department of Neurology, Royal Perth Hospital, Box X2213 GPO, Perth, WA 6001, Australia

(gjhankey@cyllene.uwa.edu.au)

John W Eikelboom consultant haematologist

Department of Haematology, Royal Perth Hospital

Competing interests: GJH and JWE have received honorariums for speaking at scientific meetings from Bristol-Myer Squibb and Sanofi-Synthelabo (manufacturer of clopidogrel). GJH has also received honorariums from Boehringer-Ingelheim (manufacturer of dypridamole) and Bayer (manufacturer of aspirin)

in patients at high risk of cardiovascular outcomes. Circulation 2002:105:1650-5.

7 Gum PA, Kottke-Marchant K, Welsh PA, White J, Topol EJ. A prospective, blinded determination of the natural history of aspirin resistance among stable patients with cardiovascular disease. J Am Coll Cardio 2003;41:961-5.

8 De Gaetano G, Cerletti C. Aspirin resistance: a revival of platelet aggregation tests? J Thromb Haemost 2003;1:2048-50.

Mosca L. C-reactive protein-to screen or not to screen? $N$ Engl J Med 2002:347:1615-7.

10 MacDonald TM, Wei L. Effect of ibuprofen on cardioprotective effect of aspirin. Lancet 2003;361:573-4.

11 Kurth T, Glynn RJ, Walker AM, Chan KA, Buring JE, Hennekens CH, et al. Inhibition of clinical benefits of aspirin on first myocardial infarction by nonsteroidal antiinflammatory drugs. Circulation 2003;108:1191-5.

\section{Management of anorexia nervosa revisited}

\section{Early intervention can help-but some cases still need tertiary inpatient care}

A recent review of outcome in anorexia nervosa pessimistically stated that the 20 th century has seen no apparent improvement ${ }^{1}$ : half the patients still never fully recover, overall mortality remains at $5 \%$, and $20 \%$ of patients stay chronically ill. Is treatment efficacy really this bad? And where does this leave the clinician?

Early intervention might be expected to exert a positive effect, and indeed this was evident in the 21 year follow up by Zipfel et al of 84 patients. ${ }^{2}$ More support comes from a recently published prevalence study of 208 patients that reported no excess mortality at 27 year follow up. ${ }^{3}$ The high rate of anorexia of $0.48 \%$ in the study on which this was based suggests that mild or early cases may have been included, though safer management of medically compromised patients might also have been a factor.

The concept of treating patients with eating disorders as hospital inpatients has its detractors, and some have claimed that treatment in general makes no difference to outcome. ${ }^{4}$ However, the study that purported to show this was flawed and could not have minority of the 220 patients received treatment which would have been generally considered to be adequate, there was no form of randomisation, and the chronicity and relatively high weights of the anorexia nervosa group made these unlikely to be representative. A multicentre, naturalistic study of 524 patients with anorexia nervosa followed for 2.5 years after admission to one of 43 hospitals in Germany has subsequently shown better outcomes with extended admission for older patients, while those who were younger and less ill benefited from a variety of treatment settings. ${ }^{6}$ So perhaps therapeutic nihilism isn't warranted.

Patients with anorexia nervosa are often seen by individual practitioners and by healthcare systems in a negative way-and not without reason. These patients' denial, hostility, and uncertain motivation for treatment; the concerned family and friends; the spectre of medical compromise; the comorbid conditions; confidentiality issues; and the challenge of behavioural containment are not for the fainthearted. The clinician's role needs to embrace engagement, exposition (having everyone tell their story), clinical evaluation and moni- 